\title{
Nível de capacitação e informação dos operadores de máquinas para a aplicação de agrotóxicos
}

\author{
Training and information level of operators machinery for pesticide application
}

\author{
André Luis Casali ${ }^{*}$ José Fernando Schlosser ${ }^{\mathrm{I}}$ Marco Antônio Gandolfo ${ }^{\text {II }}$ \\ Daniel Uhry ${ }^{I I I}$ Fabrício Azevedo Rodrigues ${ }^{I}$
}

\section{RESUMO}

O Brasil é o maior consumidor mundial de agrotóxicos. Os prejuizos causados pelo uso inadequado dos agrotóxicos ultrapassam o campo econômico, ganhando dimensão social e exigindo elevadas verbas públicas e privadas para atendimento médico hospitalar das pessoas que entram em contato, direto ou indireto, com esses produtos. O nível de conhecimento do operador das máquinas destinadas a esse fim é fundamental para diminuir esses riscos, garantindo qualidade da pulverização. Esse trabalho objetivou verificar o nivel de instrução dos operadores com relação ao manejo dos agrotóxicos e máquinas destinadas a pulverização desses insumos, na região central do Rio Grande do Sul. Observou-se que os operadores que realizaram cursos com duração maior que 20 horas, apresentaram um nivel de instrução $50 \%$ maior, se comparado aos operadores que receberam cursos com menos de 20 horas de duração. Já os operadores que realizaram cursos com duração menor que 20 horas apresentaram uma diferença de apenas quatro pontos percentuais, em relação aos operadores que não realizaram nenhum tipo de treinamento. $O$ nível de instrução dos operadores de máquinas destinadas à pulverização de agrotóxicos na Região Central do Rio Grande do Sul pode ser considerado insuficiente para o manejo correto dos processos de pulverização.

Palavras chave: agrotóxicos, contaminação ambiental, pulverizadores agrícolas.

\section{ABSTRACT}

Brazil is the world's largest consumer of pesticides. The damage caused by improper use of pesticides beyond the economic field are gaining social dimension, because they require large public funds and private hospital for medical care of people who come into direct or indirect contact with these products. The level of knowledge of the operator of the machines for this purpose is essential to minimize these risks by ensuring the quality of spray. This study aimed to determine the level of training of operators in relation to management of pesticides and spraying machines for these inputs in the central region of Rio Grande do Sul. It was observed that the operators have done courses lasting longer than 20 hours, had an education level 50\% higher when compared to operators who received courses of less than 20 hours. Operators who participated in courses lasting less than 20 hours, showed a difference of only four percent in respect of operators who have never done any training. The level of training of machine operators for the spraying of pesticides in the Central Region of Rio Grande do Sul, may be considered low in relation to the proper handling of spraying processes.

Key words: pesticides, environmental pollution, agricultural sprayers.

\section{INTRODUÇÃO}

O Brasil se destaca por ser um país com vocação para a produção de alimentos. A cada ano, os agricultores brasileiros vêm demonstrando grande capacidade competitiva em diversos setores, como o da produção de commodities agrícolas. Segundo dados da Companhia Nacional de Abastecimento (CONAB, 2013), para a safra 2012/13, a área cultivada foi de 53,31 milhões de hectares com uma produção total de aproximadamente 187,09 milhões de toneladas. Para garantir essa grande produção, muitos insumos tecnológicos estão sendo usados. Entre eles, destacam-se os mais recentes, como

\footnotetext{
'Programa de Pós-graduação em Engenharia Agrícola (PPGEA), Universidade Federal de Santa Maria (UFSM), 97105-900, Santa Maria, RS, Brasil. E-mail: andrcasali@yahoo.com.br. *Autor para correspondência.

IUniversidade Estadual do Norte do Paraná (UENP), Bandeirantes, PR, Brasil.

IIIInstituto Federal do Rio Grande do Sul (IFRS), Ibirubá, RS, Brasil. 
máquinas com alta tecnologia embarcada, plantas com elevado potencial produtivo e novos defensivos agrícolas.

A utilização dos defensivos tem por objetivo assegurar o potencial produtivo das plantas cultivadas na agricultura, "protegendo"-as de agentes externos (insetos, doenças e plantas invasoras) causadores de danos. Os agrotóxicos são classificados de acordo com sua finalidade de uso, que é definida pela ação do ingrediente ativo sobre o alvo biológico (praga). O Brasil é apontado como o maior mercado consumidor de agrotóxicos do mundo. Em 2009, o volume consumido foi de 1,06 milhões de toneladas, superando as 986,5 mil toneladas comercializadas em 2008, segundo dados do Sindicato Nacional da Indústria de Produtos para a Defesa Agrícola (SINDAG, 2010). Isso representa a utilização de 22,3 quilos de agrotóxicos por hectare cultivado na safra 2009/10, um volume 7,8\% maior do que o que teria sido utilizado em 2008/9 (20,7 quilos por hectare).

A utilização desse grande volume de agrotóxicos no nosso país acarreta riscos de contaminação ao meio ambiente e também a intoxicação do operador do maquinário. Embora fundamentais ao sistema agrícola atual, o potencial de risco ambiental dos agrotóxicos cada vez mais preocupa ambientalistas e técnicos ligados à área. Os prejuízos causados pelo uso inadequado desses produtos ultrapassam o campo econômico, ganhando dimensão social, por exigirem grandes verbas públicas e privadas para atendimento médico hospitalar das pessoas que entram em contato direto ou indireto com esses produtos. No Brasil, estima-se que existam aproximadamente 15 milhões de pessoas envolvidas no trabalho rural, dessas, em torno de 150 mil a 200 mil por ano, sofrem intoxicações agudas, devido ao contato inadequado com agrotóxicos (GARCIA \& ALVES FILHO, 2005). Muitas dessas intoxicações se devem às condições inadequadas de uso das máquinas utilizadas para pulverização dos agrotóxicos.

A manutenção dessas máquinas, dentro de limites aceitáveis de qualidade, é fundamental para garantir pulverizações seguras, do ponto de vista ambiental, e eficientes tecnicamente. Porém, alguns diagnósticos relacionados à qualidade dessas máquinas, realizados no Brasil, mostraram que grande parte delas não apresentou condições adequadas de uso, comprometendo a eficiência técnica da operação e oferecendo risco de contaminação ambiental, dos produtos agrícolas produzidos e de intoxicação do operador. DORNELLES (2008), em um estudo realizado na região Central do Rio Grande do Sul, inspecionou 84 máquinas e, dessas, segundo a metodologia utilizada pelo autor, apenas quatro estavam aptas ao uso.

Existem fatores que afetam diretamente a qualidade da aplicação e, de acordo com SCHLOSSER (2002), para se obter uma aplicação de agrotóxicos de qualidade, deve-se reunir o maior conhecimento possível sobre quatro fatores: máquina, alvo biológico, fatores climáticos e agrotóxicos. Disso, depreende-se que o nível de conhecimento do operador é fundamental para diminuir os riscos de contaminação do meio ambiente, a intoxicação do operador e garantir a qualidade da pulverização.

Em estudo de medicina ocupacional, MEKONNEN \& AGONAFIR (2002) analisaram trabalhadores que aplicavam pesticidas em cinco fazendas agrícolas na Etiópia, visando a avaliar o conhecimento, atitude e prática do uso de agrotóxicos. O estudo, utilizando aplicação de questionário padrão, mostrou que a maioria deles tinha ensino fundamental e que $93 \%$ considerava o conhecimento muito importante, enquanto apenas 3\% haviam passado por exames médicos e treinamentos. Estes autores ainda recomendaram que a educação para a segurança de pesticidas deva ser transmitida aos trabalhadores.

Este trabalho teve como objetivo a verificação do nível de conhecimento e o efeito da duração dos cursos de formação dos operadores da região central do Estado do Rio Grande do Sul, Brasil, no conhecimento das técnicas de manejo dos agrotóxicos e utilização das máquinas destinadas à pulverização desses insumos.

\section{MATERIAL E MÉTODOS}

O trabalho foi desenvolvido em 69 propriedades rurais da região central do Estado do Rio Grande do Sul, nos municípios de Cachoeira do Sul, Dilermando de Aguiar, Dona Francisca, Faxinal do Soturno, Formigueiro, Itaara, Restinga Seca, Santa Maria, São Martinho da Serra e São Sepé. A escolha das propriedades foi aleatória, com visitas sem aviso prévio, entre os meses de abril e setembro de 2011.

A metodologia de abordagem e aplicação do questionário consistiu em encontrar um operador, realizar a apresentação do projeto e a apresentação da equipe. Para a coleta de dados, foi aplicado um questionário (Tabela 1) com o intuito de verificar o nível de instrução e capacitação do operador a respeito da técnica de aplicação de agrotóxicos e o manejo adequado dos processos de pulverização. Entre os pontos abordados, estavam o grau de escolaridade, $o$ tipo de treinamento e o nível de compreensão sobre a 
Tabela 1 - Questionário aplicado aos operadores.

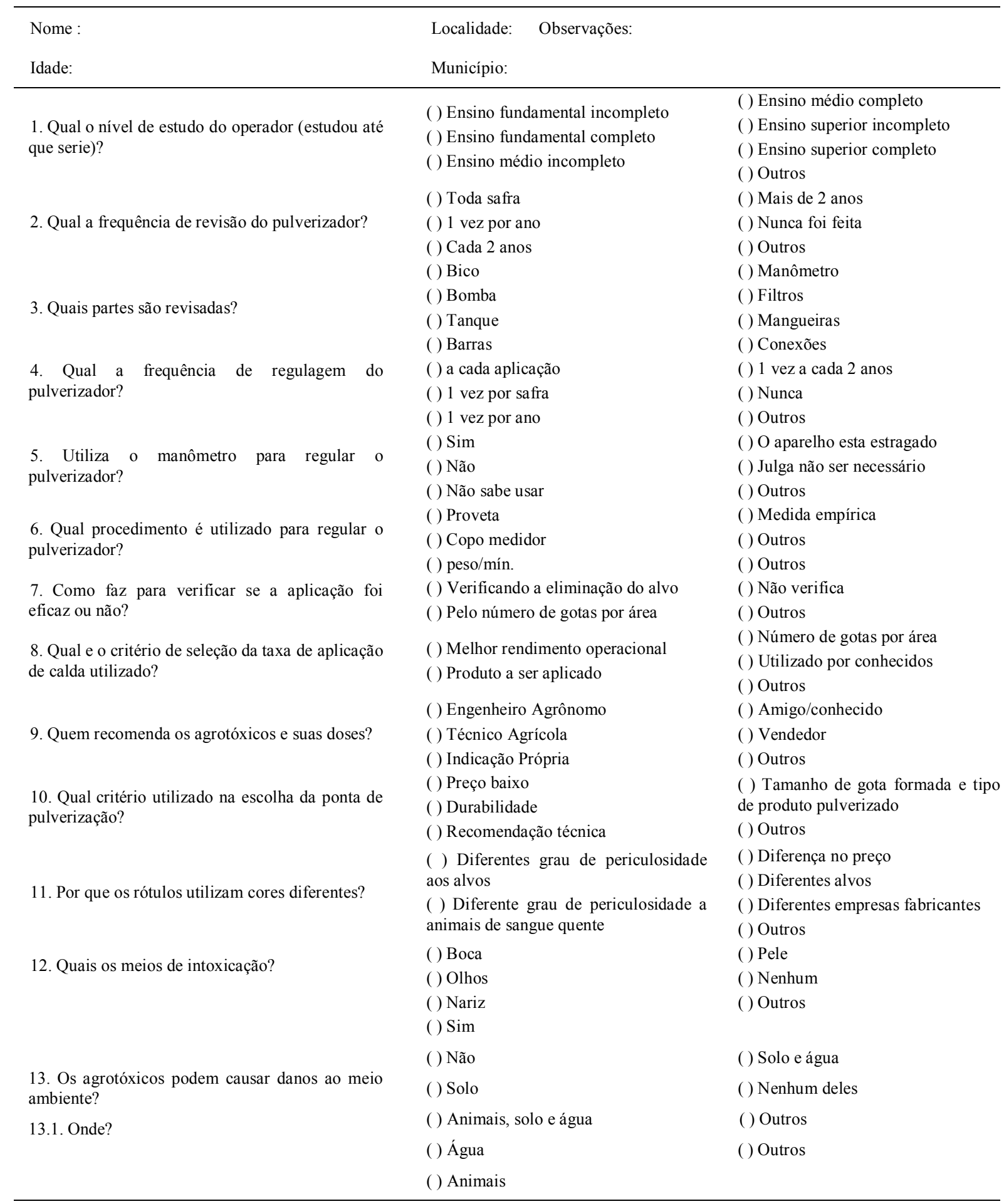

maneira correta de realizar a manutenção da máquina e de manejar adequadamente os agrotóxicos. Em relação à toxicidade dos defensivos, foi utilizada a tabela 2 como referência, para verificar o nível de conhecimento sobre a convenção de cores, utilizada nos rótulos. As respostas eram de múltipla escolha e foram apresentadas e lidas ao entrevistado para evitar possíveis situações de não entendimento por 
Tabela 2 - Classes dos agrotóxicos e suas respectivas toxicidades a animais de sangue quente.

\begin{tabular}{|c|c|c|c|}
\hline Classe toxicológica & ---------------------------Descrição------------------------- & Faixa indicativa de cor & $\begin{array}{l}\text { Quantidade capaz de matar } \\
\text { uma pessoa adulta }\end{array}$ \\
\hline I & $\begin{array}{l}\text { Extremamente tóxicos }\left(\mathrm{DL}_{50}<50 \mathrm{mg} \mathrm{kg}^{-1} \text { de peso }\right. \\
\text { vivo) }\end{array}$ & Vermelho intenso & $=5 \mathrm{mg} \mathrm{kg}^{-1}-$ algumas gotas \\
\hline II & Muito tóxicos ( $\mathrm{DL}_{50}-50$ a $500 \mathrm{mg} \mathrm{kg}^{-1}$ de peso vivo) & Amarelo intenso & 1 colher de chá \\
\hline III & $\begin{array}{l}\text { Moderadamente tóxicos }\left(\mathrm{DL}_{50}-500 \text { a } 5000 \mathrm{mg} \mathrm{kg}^{-1}\right. \\
\text { de peso vivo) }\end{array}$ & Azul intenso & 1 colher de sopa \\
\hline IV & Pouco tóxicos $\left(\mathrm{DL}_{50}>5000 \mathrm{mg} \mathrm{kg}^{-1}\right.$ de peso vivo $)$ & Verde intenso & 2 colheres de sopa \\
\hline
\end{tabular}

Adaptado de: TRAPÉ 1993.

parte dele. Em todos os casos em que o questionário foi aplicado, a mesma pessoa foi responsável por aplicá-lo, diminuindo, dessa forma, a chance de ocorrer alguma distorção na forma de apresentar os questionamentos. $\mathrm{O}$ entrevistador, logo após realizar a pergunta, fazia a leitura das opções de resposta, mostrando as alternativas ao operador, caso ele apresentasse intenção de lê-las. Ao receber a resposta, o aplicador do questionário, marcava a opção indicada pelo operador. Todos os dados originários da tabulação dos resultados foram registrados em uma planilha eletrônica no Software Microsoft Office Excel ${ }^{\circledR}$.

\section{RESULTADOS E DISCUSSÃO}

Em relação ao nível de instrução, nenhum dos operadores declarou-se analfabeto. Isto facilitou a aplicação e compreensão das questões, propostas na pesquisa, pois os respondentes, além de ouvir as perguntas, tinham também a capacidade de lê-las. $\mathrm{Na}$ distribuição do nível de escolaridade, os operadores que relataram terem completado o ensino médio representaram 38\% dos casos. Já os operadores com ensino superior completo atingiram percentual de $11 \%$ dos pesquisadores estudados, igualando esta informação à média nacional de pessoas com essa escolaridade, indicada pelo IBGE (2009). Esse elevado índice entre os operadores de pulverizadores agrícolas com ensino superior explica-se, pois, nos casos onde foram encontrados operadores com ensino superior completo, eles eram os proprietários das áreas, uma característica da região. O restante dos operadores apresentou a escolaridade indicada na figura 1 .

O reconhecimento desse nível de escolaridade não permite correlacioná-lo com o nível de conhecimento do operador em relação ao manejo correto dos agrotóxicos e também de manutenção da máquina. Dessa forma, outros questionamentos foram necessários para verificar o nível de instrução e conhecimento dos operadores com relação ao manejo correto dos defensivos.
Dos operadores que participaram da pesquisa, 37,68\% já haviam realizado algum tipo de treinamento específico relacionado ao manejo de produtos químicos fitossanitários, porém apenas $17,4 \%$ relataram ter obtido formação em cursos com duração superior a 20 horas, limite mínimo estabelecido pela Norma Regulamentadora (NR-31) do Ministério do Trabalho e Emprego, item 31.8.8.1, para que o operador esteja capacitado a manusear agrotóxicos com segurança.

Analisando as respostas registradas nos questionários, e fazendo uma comparação dos operadores que fizeram cursos com carga horária superior a 20 horas, inferior a 20 horas e os que não realizaram nenhum tipo de treinamento, observouse qu, os operadores que realizaram cursos com duração maior que 20 hora, apresentaram um nível de conhecimento $50 \%$ maior, se comparado aos operadores que receberam cursos com menos de 20 horas de duração (Figura 2). Já os operadores que realizaram cursos com duração menor que 20 horas, apresentaram uma diferença de apenas quatro pontos percentuais, em relação aos operadores que nunca realizaram nenhum tipo de treinamento. Isso comprova a necessidade de cursos com duração maior que 20 horas, para assegurar um nível de conhecimento mínimo aos operadores. Avaliando efeitos sobre os operários, CHAÍN-CASTRO et al. (1998) investigaram intoxicação por agrotóxicos em 200 trabalhadores agrícolas no México. A maioria tinha idade aproximada de 20 anos e apenas 59\% sabiam ler, diferentemente da situação encontrada neste trabalho. Poucos tinham recebido informações sobre agrotóxicos e $20 \%$ tinham sofrido intoxicação aguda por agrotóxicos, pelo menos uma vez durante a safra.

Mesmo os operadores considerados com maior nível de capacitação, não souberam responder corretamente todos os questionamentos propostos pela pesquisa (Figura 3), como foi o caso do item utilizado para regulagem dos pulverizadores. A 


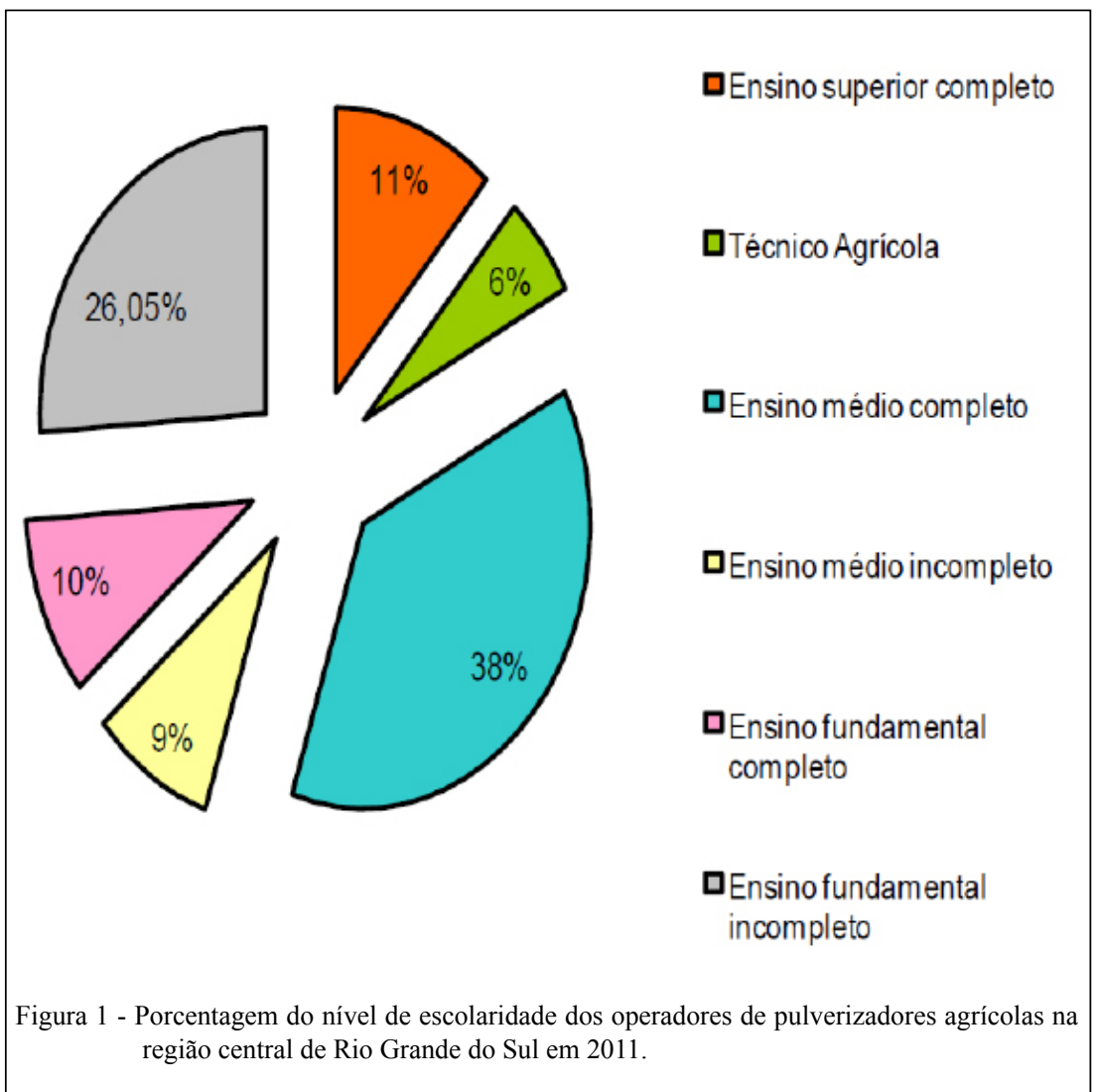

utilização de copos medidores para realização dessa atividade é considerada imprópria, porém é realizada na maioria das propriedades. Outro fator que apresentou grande percentagem de respostas incorretas de todos os operadores foi a forma de escolha da taxa de aplicação. Considerando esses dois mencionados como importantes para se obter uma confiabilidade na pulverização dos agrotóxicos,

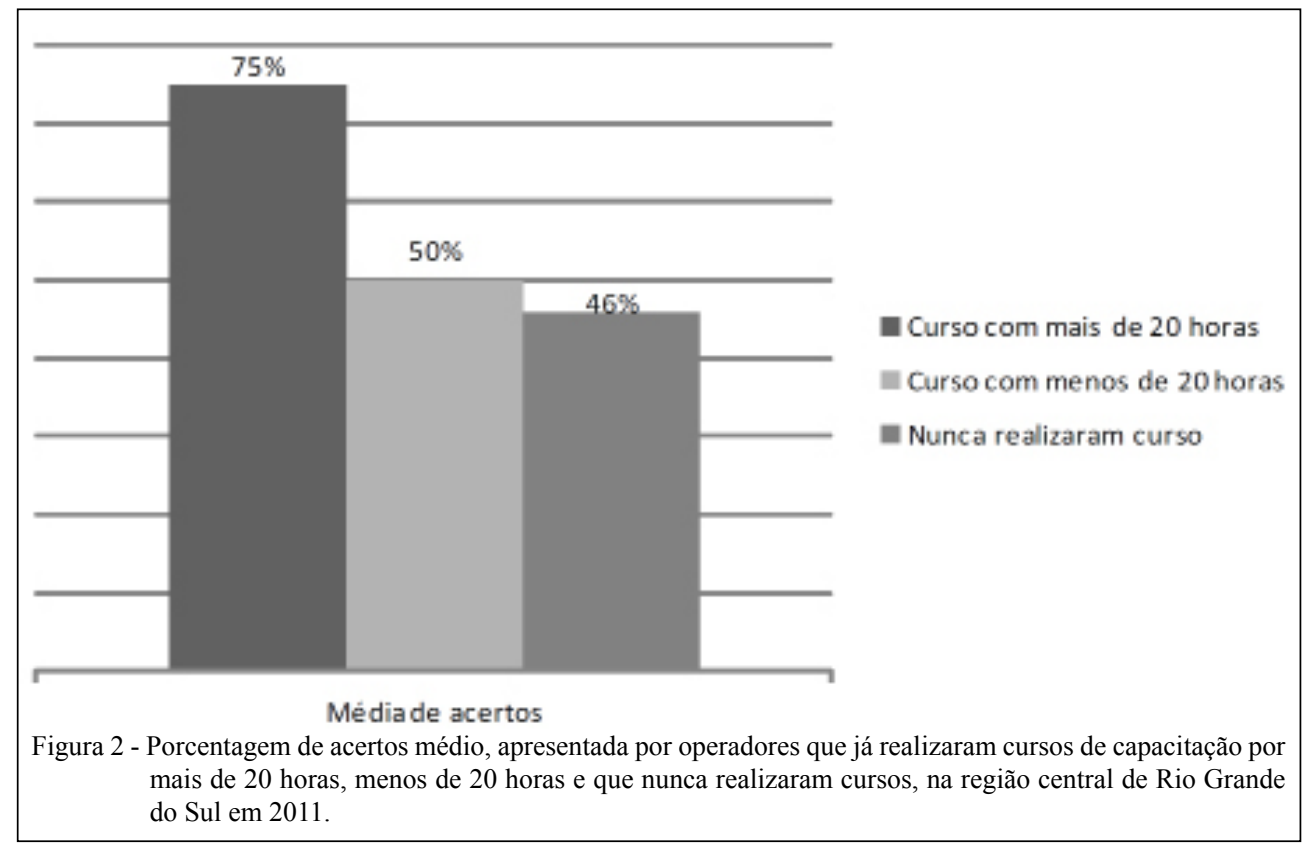

Ciência Rural, v.45, n.3, mar, 2015. 


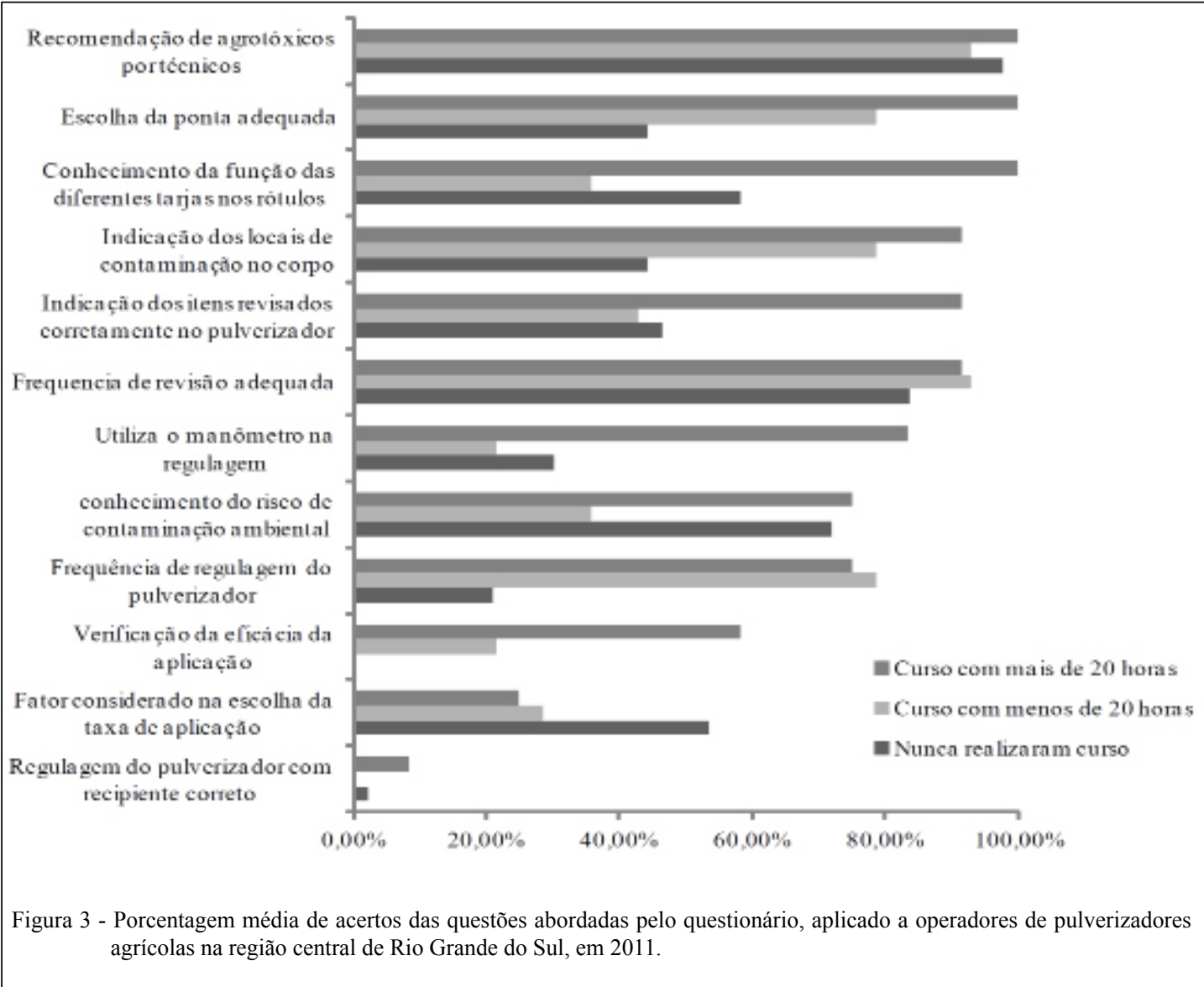

pode-se deduzir o despreparo dos operadores na condução dessa atividade, podendo proporcionar pulverizações com erros que afetam diretamente o meio ambiente e os alimentos que serão produzidos e a saúde dos próprios trabalhadores. MATTHEWS et al. (2003), em diagnóstico realizado em Camarões, na África, com aplicação de agroquímicos em programa para introduzir a adoção de requisitos mínimos para equipamentos de aplicação, já haviam concluído pela necessidade de formação contínua e disseminação de informações em todo continente.

As mais altas correlações entre nível de formação e qualidade das respostas obteve-se para as questões relacionadas à escolha da ponta de pulverização adequada, os locais do corpo em que ocorre a contaminação e consequente intoxicação e na qualificação dos itens do pulverizador que devem receber revisão e manutenção.

\section{CONCLUSÃO}

O nível de capacitação dos operadores de máquinas destinadas à pulverização de agrotóxicos na Região Central do Rio Grande do Sul pode ser considerado baixo, com referência ao manejo correto dos processos de pulverização. Operadores que realizam cursos com mais de 20 horas de duração apresentam maior nível de capacitação se comparado aos que fazem cursos com carga horária menor que 20 horas, porém, mesmo estes, ainda não possuem capacitação adequada no que diz respeito à escolha da taxa de aplicação e à regulagem dos pulverizadores. A carga horária exigida pela Norma Regulamentadora para a capacitação de aplicadores de produtos fitossanitários via pulverização parece estar minimamente adequada ao conhecimento necessário para esta função. Cargas horárias inferiores a 20 horas e a ausência de treinamento podem resultar em mau uso do equipamento e defeitos na aplicação.

\section{AGRADECIMENTOS}

Os autores agradecem ao apoio financeiro recebido da Coordenação de Aperfeiçoamento de Pessoal de Nível Superior (CAPES) e Conselho Nacional de Desenvolvimento Científico e Tecnológico (CNPq).

Ciência Rural, v.45, n.3, mar, 2015. 


\section{REFERÊNCIAS}

CHAÍN-CASTRO, T. de J. et al. Pesticide poisoning in mexican seasonal farm workers. International Journal of Occupational and Environmental Health, n.3, p.202-203.

CONAB (COMPANHIA NACIONAL DE ABASTECIMENTO). Levantamento de grãos aponta para uma safra de 195 milhões de toneladas. Brasília, 2013. p1. Disponível em: <http://www. sindag.com.br/noticia.php?News_ID=1399GazetaMercantil/ Finanças\&Mercados - Pág. 9 Gilmara Botelho>. Acesso em: 14 out. 2013.

DORNELLES, M.E. de C. Inspeção técnica de pulverizadores agrícolas no Estado do Rio Grande do Sul, 2008. $121 \mathrm{f}$ Dissertação (Mestrado em Engenharia Agrícola) - Universidade Federal de Santa Maria, Santa Maria, RS.

GARCIA, E.; ALVES FILHO, J.P. Aspectos de prevenção e controle de acidentes no trabalho com agrotóxicos. São Paulo: Fundacentro, 2005. 53p.

IBGE (INSTITUTO BRASILEIRO DE GEOGRAFIA E ESTATÍSTICA). PNAD 2009: rendimento e número de trabalhadores com carteira assinada sobem e desocupação aumenta. Disponível em: <http://www.ibge.gov.br/home/presidencia/noticias/ noticia_visualiza.php?id_noticia $=1708$ Comunicação Social setembro de $201 \overline{0}>$. Acesso em: $2 \overline{4}$ maio, 2012.

MATTHEWS, G. et al. A survey of pesticide application in Cameroon. Crop Protection, v.22, n.5, p.707-714, 2003.

MEKONNEN, Y.; AGONAFIR, T. Pesticide sprayers' knowledge, attitude and practice of pesticide use on agricultural farms of Ethiopia. Occupational Medicine, v.52, p.311, 2002.

SCHLOSSER, J.F. Tecnologia de aplicação e uso de máquinas: uso de agroquímicos. Santa Maria: UFSM, 2002. (Caderno didático - Série Técnica, Módulo 5).

SINDAG. Brasil supera EUA no uso de agroquímicos. Disponível em: <http://www.sindag.com.br/noticia.php?News ID=1399Gazet Mercantil/Finanças\&Mercados - Pág. 9 Gilmara Botelho $>$. Acesso em: $18 \mathrm{dez}, 2010$.

TRAPÉ, A.Z. O caso dos agrotóxicos. In: BUSCHINELLI, J.T. et al. (Orgs.). Isto é trabalho de gente? Vida, doença e trabalho no Brasil. Petrópolis: Vozes, 1993. p.565-593. 